\title{
Do cerebral microbleeds affect cognition in patients with symptomatic small vessel disease?
}

\author{
Kholoud Mahmoud Abdullah', Hany Mahmoud Zakieldin', Iman Mohamed Bayomy', \\ Maryse Youssef Awadallah ${ }^{2}$ and Mona Mokhtar Wahid El Din ${ }^{1 *}$
}

\begin{abstract}
Background: Dementia and cognitive impairment are becoming increasingly a major health problem, pronounced by increased life expectancy. Cerebral small vessel disease (CSVD) is among the major causes of cognitive deterioration, yet cerebral microbleeds (CMBs) which are a common association of CSVD are still not sure to be related to cognition.

Aim of the study: The study aims to determine whether the number and localization of CMBs correlate with cognition in patients with symptomatic small vessel disease (SVD), according to Montreal Cognitive Assessment (MoCA) test.

Subjects and methods: This cross-sectional study included 85 Egyptian patients with symptomatic SVD, from the neuropsychiatry clinic of the Main Suez Hospital in Suez City, in the period between February 2017 and February 2018. Subjects were classified according to CMB presence into CMB-positive and CMB-negative groups. Both groups are assessed using MRI imaging and MoCA test for cognitive function.

Results: In our study, CMBs recorded a high prevalence rate of SVD patients. Subjects with MBs were mainly males and significantly older, with higher white matter lesion volume and more lacunar infarcts. MoCA test detected significant impairment in visuospatial/executive function, attention, and total scores in CMB-positive group. Both frontal and parietal MBs showed independent association with visuospatial/executive impairment. Deep MBs in the basal ganglia were proved to be independent risk factor for attention affection.
\end{abstract}

Conclusion: Number and localization of MBs proved to be important in determining cognitive consequences. The relations with cognitive performance were mainly driven by frontal, parietal, and deep located MBs in the basal ganglia. Memory affection in frontal MBs was dependent to severe white matter intensities and lacunes.

Keywords: Cerebral microbleeds, Cognition, Small vessel disease, Dementia

\section{Introduction}

Vascular dementia and vascular cognitive impairment (VCI) main pathology \pm is cerebral ischemic small vessel disease (SVD) [1]. With the improving technology and widespread clinical use of magnetic resonance imaging (MRI), interest increased in SVD as being the most important tool for its detection and localization [2].

In addition to lacunar infarcts and leukoaraiosis as MRI signs of SVD, cerebral microbleeds (CMBs) are

\footnotetext{
* Correspondence: Wahid_el_din@hotmail.com

${ }^{1}$ Faculty of Medicine, Ain Shams University, Cairo, Egypt

Full list of author information is available at the end of the article
}

recently recognized as an important new manifestation and diagnostic marker of small vessel pathology [3]. CMBs are small, round, well-demarcated, homogeneous foci of low-signal intensity ranging $5-10 \mathrm{~mm}$ in diameter on gradient-echo (GE) T2*-weighted MRI sequences [4]. Histopathology, characterized by perivascular collections of macrophages engulfing hemosiderin, reveal an underlying vascular pathological change, hypertensive vasculopathy, and cerebral amyloid angiopathy (CAA) [5].

While WMCs and lacunar infarcts have been proved through studies to be associated with cognitive impairment [6], CMBs' impact on cognition remains unsure 
and requires more studies. They are present in about 5\% of healthy individuals without active neurological disorder with a prevalence increasing with age [7].

CMBs are commonly found in patients with ischemic or hemorrhagic stroke, $\mathrm{AD}$, and vascular dementia $(\mathrm{VaD})$ [8]. Most studies were done to examine the association of CMBs with increased risk of stroke recurrence and intracerebral hemorrhage $[9,10]$. Investigation of their presence in relation to poorer microstructural integrity of brain white matter was done to explain cognitive impairment [11].

This made the studying of CMBs in relation with cognitive performance important on patients with SVD and whether this association remained after controlling for other MRI markers of SVD. Considering other factors as number and location of CMBs will put more explanations for different vascular pathology and mechanisms of cognitive impairment in those patients [12].

\section{Aim of the study}

The aim of the study is to determine whether CMBs correlate with cognition in patients with symptomatic SVD, and if there is a relation between different locations of CMBs and their effect on cognition.

\section{Subjects and methods \\ Study population}

Eighty-five patients with SVD were recruited from the neuropsychiatry clinic of the Main Suez Hospital in Suez City according to specific criteria that must be fulfilled.

\section{Inclusion criteria}

- A clinical lacunar stroke syndrome [13] with an anatomically appropriate lacunar infarct on MRI, with or without confluent leukoaraiosis (Fazekas grade 2 or more) on MRI [14].

\section{Exclusion criteria}

- Any cause of stroke other than SVD including extra or intracranial arterial vessel stenosis $>50 \%$ or cardioembolic source

- Subcortical infarcts $>1.5 \mathrm{~cm}$ in diameter as these (striatocapsular-type infarcts) are often due to embolism or cortical infarcts

- Other major central neurological system disorders or brain injury

- Other cause for white matter disease other than SVD

- Major psychiatric disorders

- Prominent visual or hearing impairment

- Individuals with contraindications to MRI or known claustrophobia

\section{Baseline and clinical assessments}

- All SVD patients will be examined by a neurologist for full history and clinical examination.

- Registration of baseline information as age, sex, and level of education

- Assessment of cerebrovascular risk factors:

- Hypertension defined as blood pressure more than 140/90 $\mathrm{mmHg}$ and/or use of antihypertensive medications

- Diabetes mellitus defined as fasting serum glucose level $\geq 110 \mathrm{mg} / \mathrm{dL}$, postprandial more than $140 \mathrm{mg} /$ $\mathrm{dl}$, hemoglobin $\mathrm{A}_{1 \mathrm{c}}$ levels $\geq 6.5 \%$ or use of antidiabetic medication

- Hyperlipidemia defined as fasting serum total cholesterol level $\geq 200 \mathrm{mg} / \mathrm{dL}$ and/or fasting serum triglyceride levels $\geq 200 \mathrm{mg} / \mathrm{dL}$ and/or use of antihyperlipidemic agents

- Smoking status divided into current, ex-smoker, and never

- Other investigations as echocardiography and carotid duplex will be carried.

\section{Research ethics}

All patients were informed about the aim of our study and methodology, and they had agreed to participate in our research study in a written consent.

\section{MRI data analysis}

All subjects underwent a 1.5-T MRI. All images were viewed and interpreted by a consultant radiologist who was blind to the subjects' clinical data during neurological examination and cognitive function assessment.

\section{Identification of microbleeds}

CMBs are defined on GE-MRI as well-defined round focal areas of low-signal intensity and $2-10 \mathrm{~mm}$ in diameter. To measure the presence, amount, and topographical distributions of $\mathrm{CMBs}$ in each subject, the Microbleed Anatomic Rating Scale (MARS) will be used [15]. CMBs are categorized into lobar, deep, and infratentorial. Lobar is included in cortical and subcortical regions (assessed in the frontal, parietal, temporal, and occipital regions).

Deep regions include deep gray matter, including the basal ganglia, thalamus, the white matter of the corpus callosum, internal and external capsule, and the deep/ periventricular WM (DPWM). Infratentorial regions include the brain stem and the cerebellum. Deep/periventricular white matter is defined as white matter adjacent to or within $\approx 10 \mathrm{~mm}$ of the lateral ventricular margin. Symmetrical areas of basal ganglia calcification, flow voids from blood vessels, and low signals averaging from adjacent bone are discounted. 
CMBs are classified as absent, mild (1-2 microbleeds), moderate (3-10 microbleeds), or severe (> 10 microbleeds).

\section{Identification of lacunar infarcts}

Based on T1-weighted and FLAIR images, a lacune presence is defined as a cerebrospinal fluid-filled cavity, within the white matter, subcortical regions, or deep gray nuclei between 3 and $15 \mathrm{~mm}$ in diameter, with a surrounding rim of high-signal intensity following a vascular distribution.

\section{Identification of leukoaraiosis}

White matter signal hyperintensities on T2WI and FLAIR imaging was rated according to the Fazekas scale as grade 0 , no leukoaraiosis; grade 1, mild leukoaraiosis (>5 white matter hyperintensities); grade 2 , moderate confluent leukoaraiosis; and grade 3 , severe confluent leukoaraiosis.

\section{Cognitive function assessment}

The Montreal Cognitive Assessment (MoCA) questionnaire will be administered by neuropsychologists. It is a 30-point test covering seven cognitive domains: visuospatial/executive, naming, memory, attention, language, abstraction, and orientation. Cognitive impairment is indicated by a total MoCA score of $<26$. A bonus point is given to individuals with $\leq 12$ years' education [16].

The Arabic MoCA was validated and administered after its translation into Arabic then retranslation into English. The Arabic version is identical to the English version except for the two sentences used in the repetition task that have been replaced by their likes in the Arabic culture but carrying the same meaning as the English version [17].

There are characteristics in the Arabic MoCA that explain its higher sensitivity in detecting $\mathrm{MCI}$. The MoCA memory testing contains more words, fewer learning trials, and a longer delay before recall than the other tests. It can test executive functions, higher-level language abilities, and complex visuospatial processing with more numerous and demanding tasks than the other screening tests [16]. MoCA can detect approximately $90 \%$ of MCI subjects. In mild AD patients, MoCA sensitivity is $100 \%$ and specificity is considered to be $87 \%$.

\section{Statistical analysis}

Data were analyzed using Statistical Program for Social Science (SPSS) version 20.0. Quantitative data were expressed as mean \pm standard deviation (SD). Qualitative data were expressed as frequency and percentage.

\section{Results}

Association between microbleeds location and risk factors There was significant association between the presence of deep microbleeds and both hypertension ( $P$ value $0.027)$ and hyperlipidemia (0.048) as shown in Table 1. Lobar and infratentorial microbleeds did not show any significant associations with any of the risk factors.

\section{Association between antiplatelet intake and CMBs}

There was a significant association between the intake of antiplatelet medication and CMB presence $(P$ value $=$ 0.014 ), as shown in Table 2.

\section{Correlation between CMB presence and other MRI parameters of SVD}

There was a statistically significant correlation between $\mathrm{CMB}$ presence and presence of multiple lacunar infarcts ( $P$ value 0.017$)$, as well as significant correlation between severity of WMLs and number CMBs ( $P$ value 0.004 ) Table 3.

\section{Correlation between CMBs and cognitive domains according to MoCA test}

According to MoCA test for cognitive impairment, results showed statistically significant deterioration in total MoCA scores $(P$ value 0.01$)$, the visuospatial/executive sub-score $(<0.001)$, and attention $(P$ value 0.035$)$ in the CMB-positive group compared with the CMB-negative group Table 4.

\section{Relation between different $\mathrm{CMB}$ location and Montreal Cognitive Assessment (MoCA) test scores}

There was a statistical significance between MoCA test and the presence of frontal CMBs in visuospatial/executive $(P$ value $<0.001)$, memory $(P$ value $<0.001)$, and total score $(P$ value $<0.001)$ as well as a statistical significance between MoCA test and the parietal CMBs as comparing positive and negative groups in visuospatial/ executive function $(P$ value 0.016$)$ and the total score $(P$ value 0.002). MoCA test and basal ganglia CMBs show significant results in attention ( $P$ value 0.002$)$ and total score ( $P$ value 0.006$)$ as shown in Table 5.

Table 1 Cerebrovascular risk factors according to deep and non-deep located CMBs

\begin{tabular}{llllll}
\hline Risk factors & \multicolumn{2}{l}{ Deep } & & $X^{2}$ test \\
\cline { 2 - 3 } \cline { 5 - 6 } & No $(N=5)$ & Yes $(N=35)$ & & \\
\cline { 5 - 6 } & No. $(\%)$ & No. $(\%)$ & & $X^{2}$ & $P$ value \\
\hline Hypertension & $1(20.0 \%)$ & $26(74.3 \%)$ & 4.87 & 0.02 \\
Diabetes milletus & $2(40.0 \%)$ & $12(34.3 \%)$ & 0.06 & 0.80 \\
Hyperlipidemia & $1(20.0 \%)$ & $23(65.7 \%)$ & 3.81 & 0.04 \\
Smoking & $1(20.0 \%)$ & $14(40.0 \%)$ & 0.74 & 0.38 \\
\hline
\end{tabular}


Table 2 Antiplatelet intake differences between CMB-positive and CMB-negative groups

\begin{tabular}{|c|c|c|c|c|c|}
\hline \multirow[t]{2}{*}{ Medications } & & CMB-positive group & CMB-negative group & $x^{2}$ & \multirow{2}{*}{$\begin{array}{l}P \\
\text { value }\end{array}$} \\
\hline & & (\%) No & (\%) No & & \\
\hline \multirow[t]{2}{*}{ On antiplatelets } & No & $(40 \%) 16$ & $(66.7 \%) 30$ & 6.06 & 0.01 \\
\hline & Yes & $(60 \%) 24$ & (33.3\%) 15 & & \\
\hline
\end{tabular}

Data are expressed as frequency and percentage; $P$ value $<0.05$ significant; $X^{2}$ Chi square test

Relation between location of CMBs and Montreal Cognitive Assessment (MoCA) test scores after controlling other parameters

According to the statistical significance between visuospatial/executive function and the frontal CMBs, and after adjustment for personal factors, cardiovascular risk factors and MRI parameters, frontal microbleeds still showed statistical significance with visuospatial executive function impairment ( $P$ value 0.041).

While correlation between frontal microbleeds and memory showed statistically insignificant results ( $P$ value 0.176) after adjustment of MRI parameters of multiple lacunes and moderate to severe leukoaraiosis (WML grades $2-3$ ).

According to the statistical significance between visuospatial/executive function and the parietal CMBs, and after adjustment for personal factors, cardiovascular risk factors and MRI parameters, parietal microbleeds still showed statistical significance with visuospatial executive function impairment ( $P$ value 0.05 ) (Tables 6 and 7$)$.

\section{Discussion}

Whether CMBs have a role in vascular cognitive impairment or not is still controversial [17]. Different studies with different populations and patient groups have produced less consistent results according to association with cognitive dysfunction and cognitive domains affected [18].

Our study was carried out on 85 Egyptian patients with lacunar stroke. Cerebral microbleeds were frequently observed in 40 patients (47\%), which is nearly half of all patients. This is highly consistent with the previous studies of Zhang et al. [19] that studied 85 patients; 35 (41.2\%) had cerebral microbleeds. They were more often in mixed and deep brain regions than in the cerebral lobes. There was a significant correlation between cerebral microbleed severity and MoCA score [20]. CMBs were present in 46 (39.7\%) patients. CMB number was weakly associated with executive dysfunction. This study did not find association of CMBs with other cognitive domains, including processing speed. On the other hand, in other studies [21], patients with MBs (14.2\%) were significantly older, had a higher white matter lesion volume, and more lacunar infarcts. Number of MBs were related to global cognitive function and attention. The relations with cognitive performance were mainly determined by frontal, temporal, and deep located MBs [12]. Nine hundred fifty-nine subjects were studied. MBs were found in $10.4 \%$ of the subjects; strictly lobar, but not deep or infratentorial, CMBs are associated with changes in cognitive function, especially in visuospatial/executive functions. Cerebral amyloid angiopathy in this study suggested to be the underlying pathology associated with CMB-related cognitive impairment.

CMBs constituted much less percentage of the population studied. This may be due to the difference in samples' sizes and nature of these studies being both cohort. It also included variable population groups than small vessel disease [12].

CMBs were detected in older patients, and this was proven as a consistent risk factor across different studies $[12,22]$. They attributed the effect of age on accumulation of other vascular risk factors that lead to $\mathrm{CMB}$ pathology.

Male gender showed a significant association with microbleed presence. This was consistent with the previous studies $[10,23]$ that proved being the male gender as one of the risk factors of CMB presence. This association went under research, and was hypothesized as vascular risk factors being more common in males and reference to the role of apolipoprotein APOE4 allele interaction with sex differences and amyloid $\beta$-peptide $(\mathrm{A} \beta)$ levels [24].

Still, there were some studies [12, 20] that were inconsistent with ours and showed insignificant association

Table 3 MRI parameters compared between CMB-positive and CMB-negative groups

\begin{tabular}{lllll}
\hline MRI parameters & CMB-positive group & CMB-negative group & $X^{2}$ & $P$ \\
& No (\%) & No (\%) & 7.48 & 0.01 \\
\hline Lacunar $>1$ & $15(37.5 \%)$ & $8(17.8 \%)$ & 10.79 & $0.004^{*}$ \\
WML severity 2-3 & $24(60 \%)$ & $13(28.9 \%)$ & \\
\hline
\end{tabular}

Data are expressed as frequency and percentage

$X^{2}$ Chi square test

${ }^{*} P$ value $>0.05$ non-significant 
Table 4 Comparison between groups according to MoCA test

\begin{tabular}{|c|c|c|c|c|c|}
\hline MoCA test & & CMB-positive & CMB-negative & $x^{2}$ & $P$ value \\
\hline \multirow[t]{2}{*}{ Visuospatial/executive } & Mean \pm SD & $3.03 \pm 1.82$ & $4.18 \pm 0.81$ & 14.82 & $<0.001$ \\
\hline & Range & $0-5$ & $2-5$ & & \\
\hline \multirow[t]{2}{*}{ Naming } & Mean \pm SD & $2.75 \pm 0.44$ & $2.82 \pm 0.39$ & 0.65 & 0.42 \\
\hline & Range & $2-3$ & $2-3$ & & \\
\hline \multirow[t]{2}{*}{ Attention } & Mean \pm SD & $3.03 \pm 1.44$ & $4.27 \pm 0.91$ & 4.87 & 0.03 \\
\hline & Range & $1-6$ & $2-6$ & & \\
\hline \multirow[t]{2}{*}{ Language } & Mean \pm SD & $2.85 \pm 0.43$ & $2.87 \pm 0.50$ & 0.02 & 0.87 \\
\hline & Range & $1-3$ & $2-3$ & & \\
\hline \multirow[t]{2}{*}{ Abstraction } & Mean \pm SD & $1.55 \pm 0.55$ & $1.56 \pm 0.50$ & 0.002 & 0.96 \\
\hline & Range & $0-2$ & $1-2$ & & \\
\hline \multirow[t]{2}{*}{ Memory } & Mean \pm SD & $3.20 \pm 1.24$ & $3.78 \pm 0.77$ & 1.80 & 0.10 \\
\hline & Range & $1-5$ & $2-5$ & & \\
\hline \multirow[t]{2}{*}{ Orientation } & Mean \pm SD & $5.48 \pm 0.55$ & $5.56 \pm 0.59$ & 0.42 & 0.51 \\
\hline & Range & $4-6$ & $4-6$ & & \\
\hline \multirow[t]{2}{*}{ Total MoCA test } & Mean \pm SD & $23.48 \pm 4.75$ & $25.51 \pm 2.45$ & 6.37 & 0.01 \\
\hline & Range & $14-30$ & $18-30$ & & \\
\hline
\end{tabular}

between microbleeds and sex, though the high incidence is in males, but were not statistically significant.

Hypertension was considered a statistically significant risk factor for the development of CMBs. Other studies $[23,25]$ did not only identify hypertension as a risk factor, but also studied the attribution of deep and infratentorial microbleeds to hypertensive microangiopathy mechanism which is consistent with ours.

Chung and colleagues in 2016 did not find any correlation between CMBs and hypertension. This may be explained as studying lobar CMBs, with suggested CAA as an underlying pathology rather than hypertensive arteriopathy mechanism [12]. Correlation between hyperlipidemia and MBs was significant. Such an attribution was consistent with the other studies $[23,26]$ that studied the effect of accumulated risk factors on CMB incidence. The significant association between hyperlipidemia and patients with deep CMBs was also consistent with other studies $[27,28]$ that attributed cerebrovascular risk factors to deep located CMBs.
CMBs were more frequent in antiplatelet users than in non-antiplatelet users as found by Pasquini et al. [29]. A meta-analysis on the relationship between antiplatelet therapy and CMBs found that antiplatelet therapy was significantly associated with presence of CMBs in patients with stroke but not in stroke-free individuals [29]. A more recent meta-analysis [30] that included 37 studies did not just clarify the increased risk of CMBs on antiplatelet therapy; moreover, they related their use to the association with strictly lobar microbleeds rather than deep/infratentorial microbleeds.

Fewer studies [31] did not find any correlation between regular antiplatelet use and CMB presence. However, it did not rule out the risk of $\mathrm{CMB}$ formation and $\mathrm{ICH}$, but rather, recommended antiplatelet justification and controlled other risk factors in the cases of ischemic cerebrovascular diseases. After all, it described having many potential limitations specially their small study population and a high selection bias, yet, the use of single vs. double antiplatelet did not record statistical

Table 5 Partial correlation between MoCA test cognitive indices

\begin{tabular}{llllllllll}
\hline & & Visuospatial/executive & Naming & Attention & Language & Abstraction & Memory & Orientation & Total \\
\hline Frontal & $r$ & -0.57 & -0.20 & -0.27 & -0.22 & -0.22 & -0.49 & -0.16 & -0.53 \\
& $p$ & $<0.001$ & 0.211 & 0.080 & 0.173 & 0.163 & $<0.001$ & 0.106 & $<0.001$ \\
Parietal & $r$ & -0.47 & -0.23 & -0.27 & -0.17 & -0.26 & -0.14 & -0.17 & -0.47 \\
& $p$ & 0.01 & 0.14 & 0.08 & 0.29 & 0.10 & 0.24 & 0.46 & 0.002 \\
\multirow{2}{*}{ Basal ganglia } & $r$ & -0.28 & -0.30 & -0.46 & -0.13 & -0.02 & -0.26 & -0.16 & -0.42 \\
& $p$ & 0.07 & 0.05 & 0.002 & 0.40 & 0.86 & 0.09 & 0.47 & 0.006 \\
\hline
\end{tabular}

$r$ Pearson correlation coefficient 
Table 6 Correlations between frontal CMBs and cognitive indices after controlling different parameters

\begin{tabular}{|c|c|c|c|}
\hline & \multicolumn{3}{|l|}{ Frontal } \\
\hline & $P$ value* & $P$ value & $P$ value \\
\hline Visuospatial/executive & $-0.70(<0.001)$ & $-0.58(<0.001)$ & $-0.33(0.04)$ \\
\hline Memory & $-0.48(0.03)$ & $-0.35(0.03)$ & $-0.25(0.17)$ \\
\hline \multicolumn{4}{|c|}{$\begin{array}{l}{ }^{*} P \text { value: Adjusted for age, sex, and years of education } \\
P \text { value: Adjusted for age, sex, years of education, and the presence of } \\
\text { cardiovascular risk factors (hypertension, diabetes mellitus, smoking, } \\
\text { and hyperlipidemia) } \\
{ }^{¥} P \text { value: Adjusted for age, sex, years of education, the presence of } \\
\text { cardiovascular risk factors, the lacunar }>1 \text {, and the severity of white matter } \\
\text { lesions (WML severity } 2-3 \text { ) }\end{array}$} \\
\hline
\end{tabular}

significance in CMB-positive group consistently similar to other studies [32].

As regarding MRI parameters, a strong correlation between $\mathrm{CMB}$ presence and the presence of other cerebral SVDs was recorded. Subjects with CMBs had significantly higher number of lacunar infarcts and more severe WMLs similar to previous studies [12, 20, 21]. All are markers of small vessel disease pathology that with their progression the incidence of microbleeds increases.

Correlation between CMB presence and cognitive impairment was seen as significant deterioration in both visuospatial/executive function and attention with a decrease in total MoCA score. Association of microbleeds with cognitive decline was consistently reported by previous studies. These results explicitly relate CMB lesions to global and executive dysfunction whether through their direct effect on microstructure brain damage or as a reflection of the underlying vascular pathology in patients with SVD [4].

A controversial study reported that the presence and number of CMBs were not associated with cognitive dysfunction in patients with lacunar stroke and leukoaraiosis [20]. It referred to other SVD markers as having a masking effect on the cognitive deterioration. Also, a cohort study [33] studied the long-term cognitive outcome of CMBs in patients with TIA and minor strokes after 5 years and denied any association with cognitive impairment. Unfortunately, it had a main weak point where $15 \%$ of the study sample were examined for the

Table 7 Correlations between parietal CMBs and cognitive indices after controlling different parameters

\begin{tabular}{|c|c|c|c|}
\hline & \multicolumn{3}{|l|}{ Parietal } \\
\hline & $P$ value* & $P$ value & $P$ value $^{¥}$ \\
\hline Visuospatial/executive & $-0.48(<0.001)$ & $-0.42(<0.001)$ & $-0.32(0.05)$ \\
\hline \multicolumn{4}{|c|}{$\begin{array}{l}{ }^{*} P \text { value: Adjusted for age, sex, and years of education } \\
P \text { value: Adjusted for age, sex, years of education, and the presence of } \\
\text { cardiovascular risk factors (hypertension, diabetes mellitus, smoking, } \\
\text { and hyperlipidemia) } \\
{ }^{¥} P \text { value: Adjusted for age, sex, years of education, the presence of } \\
\text { cardiovascular risk factors, the lacunar }>1 \text {, and the severity of white matter } \\
\text { lesions (WML severity } 2-3 \text { ) }\end{array}$} \\
\hline
\end{tabular}

cognitive status through the short version of the Informant Questionnaire on Cognitive Decline in the Elderly (IQCODE, Dutch version) that was obtained from a close relative and not personally performed. This can be opposed by another cohort study [10], which was performed on stroke clinic patients, with much similar population and had results consistent with ours regarding cognitive deterioration, proving CMBs' relevance to long-term cognitive outcome.

According to $\mathrm{CMB}$ location, both frontal and parietal MBs recorded significant associations with visuospatial/ executive dysfunction, independent to lacunes and WMCs and other factors. This was consistent with other studies $[10,12]$ that studied executive function impairment on strictly lobar CMB subjects. These studies explained such association to CAA pathology in these brain regions.

Various brain areas are involved in the visuospatial executive function; visual perception in the parieto-occipital lobe, planning in the frontal lobe, and integration of visual and fine motor sequences in the fronto-parieto-occipital cortices. Visuoperceptual skills requires mental flexibility which mainly rely on frontal lobe function, while selfinitiated clock drawing requires intact visuoconstructive skills which are mainly represented in the parietal lobe. This process of contribution of the parieto-frontal cortical networks to integrate visuospatial elements with motor control was detected by functional MRI [34].

Patel and colleagues in 2013 related the association of frontal CMBs to executive dysfunction only under a threshold effect of 9 or more CMBs in small vessel disease patients [20]. Whereas, other study [17] found that individuals with a strictly lobar distribution of CMBs did not have any impaired executive function. However, the executive function decline that was detected between CMB-positive and CMV-negative groups was explained as being driven by deep cerebral SVD (as BG MBs) rather than being due to lobar MBs. However, this study had limitations of the small sub-sample sizes.

Frontal lobe MBs also recorded an association with memory affection. However, it lost its significance with regression analysis to give similar results with previous study. The association between working memory and frontal lobe MBs in a population of symptomatic small vessel disease relationship became non-significant when controlling of other MRI parameters. It may be explained as being highly memory-dependent on WMLs and brain atrophy [20].

Inconsistent with our results, some other studies identified CMBs in patients followed in memory clinics $[23,35]$. They showed high prevalence of CMBs in memory clinics and Alzheimer's disease patients, adding more evidence in vascular cognitive impairment role. Unfortunately, memory affection was studied as a point of significance between 
both CMB-positive and CMB-negative groups in general without ruling out other MRI parameters as small vessel disease effect.

In the current study, the deep MBs were frequently located in the BG, recording significant association with global cognitive decline and with attention affection in particular. It remained significant after controlling of SVD markers to be consistent with other studies $[28,36]$. They offered some explanations about cognitive dysfunction through attribution of the cognitive deficits in CMBs to impairments in attention and calculation in the early stages of Parkinson's disease, thus reflecting involvement of the BG and frontal-subcortical circuits. CMBs in the BG may cause primary or secondary damage to cholinergic pathways in the cortical-BG circuits [37].

There were still some studies inconsistent with ours according to the involvement of BG CMBs in cognitive dysfunction [10]. Although including a large population, it classified subjects according to CMB location into only two groups which were strictly lobar and deep/infratentorial, with the last accounting only $9 \%$ of the sample size [12].

According to the other deep located and infratentorial CMBs, our study did not show any association between their presence and cognition affection at any of MoCA test domains. This was quite consistent with the number of studies $[10,20]$ that showed no significant associations with their presence and cognitive dysfunction.

However, thalamic CMBs in particular had some debate on its involvement in the development of cognitive dysfunction. Yakushiji and colleagues in 2008 showed an association between the presence of thalamic MBs and reduced both total and orientation scores in the MMSE test. It was explained as being the thalamus part of a neuronal network integrally involved in cognitive function. Other studies were neuroimaging studies on patients with CADASIL, which showed associations between microstructural alterations in the thalamus and low MMSE score [28].

\section{Conclusion}

Location of MBs proved to be important in determining cognitive consequences. Both frontal and parietal MBs showed independent association with visuospatial/executive impairment, while frontal MBs showed involvement in memory affection that was dependent to severe white matter intensities and lacunes. Deep MBs in the basal ganglia was proved to be an independent risk factor for attention affection.

\section{Limitations of the study}

The use of antiplatelets is an important confounding factor for the presence of CMBs that needs to be studied and addressed. Yet, the data necessary to adjust our results were not collected, and this is considered a limitation that could be investigated in other research.

\section{Abbreviations}

CAA: Cerebral amyloid angiopathy; CMBs: Cerebral microbleeds;

cSVD: Cerebral small vessel disease; DPWM: Deep periventricular white matter; MARS: Microbleed Anatomical Rating Scale; MoCA: Montreal

Cognitive Assessment; MRI: Magnetic resonance imaging; SVD: Small vessel disease; VaD: Vascular dementia; VCl: Vascular cognitive impairment; WMC: White matter changes

\section{Acknowledgements}

Not applicable.

Authors' contributions

All authors read and approved the final manuscript.

Funding

Self-financing.

Availability of data and materials

All data and materials that support the findings in this manuscript are available. Please contact the author for data requests.

\section{Ethics approval and consent to participate}

The procedures followed were in accordance with the ethical standards of the responsible committee on human experimentation and with the principles of Helsinki Declaration. Informed consent was obtained from all participants and ethical committee permission from the Neuropsychiatry Department of Ain Shams University at February 2, 2018.

Consent for publication

Not applicable.

\section{Competing interests}

The authors declare that they have no competing interests.

\section{Author details}

${ }^{1}$ Faculty of Medicine, Ain Shams University, Cairo, Egypt. ${ }^{2}$ Cairo University, Giza, Egypt.

Received: 7 May 2019 Accepted: 27 December 2019

Published online: 09 January 2020

References

1. Pantoni L. Cerebral small vessel disease: from pathogenesis and clinical characteristics to therapeutic challenges. Lancet Neurol. 2010;9:689-701.

2. Gorelick PB, Scuteri A, Black SE, et al. Vascular contributions to cognitive impairment and dementia: a statement for healthcare professionals from the American Heart Association/American Stroke Association. Stroke. 2011; 42:2672-713.

3. Charidimou A, Werring DJ. Cerebral microbleeds: detection, mechanisms and clinical challenges. Future Neurol. 2011;6:587-611.

4. Werring DJ, Frazer DW, Coward $L$, et al. Cognitive dysfunction in patients with cerebral microbleeds on T2*-weighted gradient-echo MRI. Brain. 2004; 127:2265-75.

5. Warach S, Greenberg SM, Vernooij MW, et al. Cerebral microbleeds: a guide to detection and interpretation. Lancet Neurol. 2009:8:165-74.

6. Sigurdsson S, Qiu C, Cotch MF, et al. Cerebral microbleeds, retinopathy, and dementia: the 13 AGES-reykjavik study. Neurology. 2010;75:2221-8.

7. Vernooij MW, van der Lugt A, Ikram MA, et al. Prevalence and risk factors of cerebral microbleeds: the Rotterdam scan study. Neurology. 2008;70:1208-14.

8. Kester MI, Goos JD, Teunissen CE, et al. Associations between cerebral small vessel disease and Alzheimer disease pathology as measured by cerebrospinal fluid biomarkers. JAMA Neurol. 2014;71:855-62.

9. Imaizumi T, Inamura S, Nomura $T$, et al. The severity of white matter lesions possibly influences stroke recurrence in patients with histories of lacunar infarctions. J Stroke Cerebrovasc Dis. 2015;24:2154-60.

10. Gregoire SM, Scheffler G, Jäger HR, et al. Strictly lobar microbleeds are associated with executive impairment in patients with ischemic stroke or transient ischemic attack. Stroke. 2013;44:1267-72.

11. Akoudad S, de Groot M, Koudstaal PJ, et al. Cerebral microbleeds are related to loss of white matter structural integrity. Neurology. 2013;81:1930-7. 
12. Chung CP, Chou KH, Chen WT, et al. Strictly lobar cerebral microbleeds are associated with cognitive impairment. Stroke. 2016;47:2497-502.

13. Bamford J, Sandercock $P$, Jones $L$, et al. The natural history of lacunar infarction: the Oxfordshire Community Stroke Project. Stroke. 1987:18:545-51.

14. Fazekas F, Chawluk JB, Alavi A, et al. MR signal abnormalities at $1.5 \mathrm{~T}$ in Alzheimer's dementia and normal aging. AJR Am J Roentgenol. 1987; 149:351-6.

15. Gregoire SM, Chaudhary UJ, Brown MM, et al. The Microbleed Anatomical Rating Scale (MARS): reliability of a tool to map brain microbleeds. Neurology. 2009;73:1759-66.

16. Rahman TTA, El Gaafary MM. Montreal Cognitive Assessment Arabic version: reliability and validity prevalence of mild cognitive impairment among elderly attending geriatric clubs in Cairo. Geriatr Gerontol Int. 2009;9:54-61.

17. Paradise M, Seruga A, Crawford JD, et al. The relationship of cerebral microbleeds to cognition and incident dementia in non-demented older individuals. Brain Imaging Behav. 2018;84(8).

18. Boyano I, Ramos A, López-Alvarez J, et al. Cerebral microbleeds in advanced dementia: clinical and pathological correlates. Am J Alzheimers Dis Other Demen. 2018;18(4):59-78.

19. Zhang M, Chen M, Wang Q, et al. Relationship between cerebral microbleeds and cognitive function in lacunar infarct. J Int Med Res. 2013; 41:347-55.

20. Patel B, Lawrence AJ, Chung AW, et al. Cerebral microbleeds and cognition in patients with symptomatic small vessel disease. Stroke. 2013;44:356-61.

21. Anouk GW, van Norden W, Heleen AC, et al. Frontal and temporal microbleeds are related to cognitive function: the Radboud University Nijmegen Diffusion Tensor and Magnetic Resonance Cohort (RUN DMC) Study. Stroke. 2011;42:3382-6.

22. Matsuyama H, li Y, Maeda M, et al. Background and distribution of lobar microbleeds in cognitive dysfunction. Brain Behav. 2017;7(11):e00856.

23. Shams S, Martola J, Granberg T, et al. Cerebral microbleeds: different prevalence, topography, and risk factors depending on dementia diagnosis: the Karolinska Imaging Dementia Study. AJNR Am J Neuroradiol. 2015;36:661-6.

24. Cacciottolo M, Christensen A, Moser A, et al. The APOE4 allele shows opposite sex bias in microbleeds and Alzheimer's disease of humans and mice. Neurobiol Aging. 2016;37:47-57.

25. Yubi T, Hata J, Ohara T, et al. Prevalence of and risk factors for cerebral microbleeds in a general Japanese elderly community. Am Acad Neurol. 2018;4(8):78-98.

26. Ding J, Sigurdsson S, Garcia M, et al. Risk factors associated with incident cerebral microbleeds according to location in older people: the Age, Gene/Environment Susceptibility (AGES)-Reykjavik Study. JAMA Neurol. 2015;72:682-8.

27. Poels MM, Ikram MA, van der Lugt A, et al. Incidence of cerebral microbleeds in the general population: the Rotterdam Scan Study. Stroke. 2011;42:656-61.

28. Yakushiji Y, Noguchi T, Hara M, et al. Distributional impact of brain microbleeds on global cognitive function in adults without neurological disorder. Stroke. 2012;43:1800-5.

29. Pasquini M, Benedictus MR, Boulouis G, et al. Incident cerebral microbleeds in a cohort of intracerebral hemorrhage. Stroke. 2016;47:689-94.

30. Qiu J, Ye H, Wang J, et al. Antiplatelet therapy, cerebral microbleeds, and intracerebral hemorrhage: a meta-analysis. Stroke. 2018;49:00.

31. Nishikawa T, Ueba T, Kajiwara M, et al. Cerebral microbleeds in patients with intracerebral hemorrhage are associated with previous cerebrovascular diseases and white matter hyperintensity, but not with regular use of antiplatelet agents. Neurologia Medico Chirurgica. 2009;49:333-9.

32. Wang Z, Wong A, Liu W, et al. Cerebral microbleeds and cognitive function in ischemic stroke or transient ischemic attack patients. Dement Geriatr Cogn Disord. 2015:40:130-6.

33. Brundel M, Kwa VI, Bouvy WH, et al. Cerebral microbleeds are not associated with long-term cognitive outcome in patients with transient ischemic attack or minor stroke. Cerebrovasc Dis. 2014;37:195-202.

34. Sánchez-Cubillo I, Periáñez JA, Adrover-Roig D, et al. Construct validity of the Trail Making Test: role of task-switching, working memory, inhibition/ interference control, and visuomotor abilities. J Int Neuropsychol Soc. 2009; 15:438-50.

35. Goos JD, Henneman WJ, Sluimer JD, et al. Incidence of cerebral microbleeds: a longitudinal study in a memory clinic population. Neurology. 2010;74:1954-60.
36. Van Norden AG, van den Berg HA, de Laat KF, et al. Frontal and temporal microbleeds are related to cognitive function: the Radboud University Nijmegen Diffusion Tensor and Magnetic Resonance Cohort (RUN DMC) Study. Stroke. 2011;42:3382-6.

37. Bronnick K, Emre M, Lane R, et al. Profile of cognitive impairment in dementia associated with Parkinson's disease compared with Alzheimer's disease. J Neurol Neurosurg Psychiatry. 2007;78:1064-8.

\section{Publisher's Note}

Springer Nature remains neutral with regard to jurisdictional claims in published maps and institutional affiliations.

\section{Submit your manuscript to a SpringerOpen ${ }^{\circ}$ journal and benefit from:}

- Convenient online submission

- Rigorous peer review

- Open access: articles freely available online

High visibility within the field

- Retaining the copyright to your article

Submit your next manuscript at $\boldsymbol{\sim}$ springeropen.com 